\title{
Medium to long term intraocular pressure control following small flap trabeculectomy (microtrabeculectomy) in relatively low risk eyes
}

\author{
S A Vernon, C Gorman, H J Zambarakji
}

\begin{abstract}
Aim-To determine the long term efficacy of small flap trabeculectomy (microtrabeculectomy) in terms of intraocular pressure (IOP) control in relatively low risk eyes.

Method-A review of a case series of small flap trabeculectomy procedures performed on 36 eyes from 36 patients with a minimum follow up of 24 months (mean 50.8).

Results-The mean (SD) intraocular pressures at presentation and preoperatively were $33.7(7.5)$ and 24.6 (4.5) $\mathrm{mm}$ Hg. At 6 months, 1, 2, 3, 4, 5, and 6 years the mean (n, SD) IOPs (mm Hg) of those eyes followed to each time point were $\mathbf{1 1 . 9}$ $(36,4.6), 12.6(36,4.7), 13.2(36,4.6), 13.7$ $(29,4.1), 13.2(22,4.0), 12.7(15,4.8)$, and $12.3(8,4.7)$ respectively. There was no significant difference in IOP levels at any of the analysis points by one way ANOVA. Kaplan-Meier analysis revealed survival rates of $80 \%$ at 4 years and $75 \%$ at 5 years when any postoperative IOP $>20 \mathrm{~mm} \mathrm{Hg}$ is considered a failure, and $50 \%$ at 6 years when any IOP $>15 \mathrm{~mm} \mathrm{Hg}$ is classed as a failure.

Conclusion-Small flap trabeculectomy (microtrabeculectomy) is effective at reducing IOP in low risk glaucoma eyes with IOP control similar to previous reports of filtering surgery utilising larger scleral trapdoors.

(Br F Ophthalmol 1998;82:1383-1386)
\end{abstract}

Trabeculectomy, introduced by Cairns ${ }^{1}$ in 1968, has become the gold standard filtering procedure for many eyes with glaucoma. A number of variations on the original technique have been described. These have included changing the size ${ }^{2}$ shape, ${ }^{3}$ and position ${ }^{4}$ of the sclerostomy and trapdoor, limbal, or fornix based conjunctival incisions, ${ }^{5}$ and altering the method of performing the sclerostomy by trephination, ${ }^{6}$ sclerectomy, ${ }^{7}$ and the use of a scleral punch. ${ }^{8}$ That no one simple procedure has been universally accepted implies that, in terms of IOP control, none has a significant advantage over any of the others. It is generally accepted that preoperative risk factors are the most important determinants of surgical success, and the use of adjunctive antimetabolite treatment in high risk cases has gained popularity over the last decade. ${ }^{9}{ }^{10}$

Early postoperative pressure control following small flap trabeculectomy (microtrab- eculectomy) has been shown to be comparable with published series for conventional trabeculectomy. ${ }^{11}$ The aim of this study was to report on the medium to long term IOP control following this procedure in a cohort of eyes at relatively low risk for surgical failure.

\section{Materials and methods}

All eyes which had undergone small flap trabeculectomy in the period January 1991January 1995 with a minimum follow up of 2 years were enrolled in the study. (All filtering procedures performed under the care of the lead clinician (SAV) during this time period were of the small flap design.) Excluded were eyes whose fellow was already enrolled, eyes from black patients, and those eyes in which antimetabolite supplementation had been used. (We use antimetabolite supplementation routinely for eyes with uveitis, rubeotic eyes, and eyes which have had previous intraocular surgery.) Demographic data and data on IOP levels, antiglaucoma medications, surgical complications and subsequent surgery, were acquired from a detailed examination of the case notes and entered onto a microcomputer database. All IOP recordings were made between 09:00 and 12:30 and were performed using slit lamp based Goldmann tonometry by ophthalmologists with regularly calibrated tonometers. Statistical analysis was performed using the mUltistat (Biosoft, Cambridge) statistical package and Kaplan-Meier analysis via sPSs version 6.0 .

"Small flap" trabeculectomy (microtrabeculectomy) is a much scaled down modification of the "full size" procedure, with the intention being to perform a filtering operation that is less disruptive to ocular tissues. Full details of the surgical technique have been described elsewhere. ${ }^{11}$ Briefly, a limbus based conjunctival flap is fashioned commencing 4 $\mathrm{mm}$ from and exposing the limbus. A $2 \mathrm{~mm} \times$ $2 \mathrm{~mm}$ scleral trap door is constructed and an anteriorly sited $0.75 \mathrm{~mm}$ diameter internal sclerostomy is achieved with a Kelly punch (Storz). A small basal peripheral iridectomy is followed by two 10/0 nylon scleral trapdoor sutures and a running 10/0 gauge suture of choice to the conjunctiva. Balanced salt solution (Alcon) and air are then injected into the anterior chamber via a paracentesis performed at the start of surgery. Postoperative medications routinely employed are atropine $1 \%$ drops three times a day and betamethasone $0.1 \%$ with neomycin sulphate $0.5 \%$ four times a day for the first week. At the 1 week review 
Table 1 Medications used preoperatively and at the last recorded postoperative visit

\begin{tabular}{lcl}
\hline & Preop & \multicolumn{1}{l}{ Postop } \\
\hline$\beta$ blocker only & $17(47 \%)$ & $5(13 \%)$ \\
Pilocarpine only & $1(3 \%)$ & $0(0 \%)$ \\
Dorzolamide only & $0(0 \%)$ & $1(3 \%)$ \\
$\beta$ blocker + pilocarpine & $11(30 \%)$ & $0(0 \%)$ \\
$\beta$ blocker + dipivefrin & $4(11 \%)$ & $0(0 \%)$ \\
$\beta$ blocker and dorzolamide & $0(0 \%)$ & $1(3 \%)$ \\
Oral acetazolamide and $\beta$ blocker & $2(6 \%)$ & $0(0 \%)$ \\
Latanoprost only & $0(0 \%)$ & $1(3 \%)$ \\
Nil & $1(3 \%)$ & $28(78 \%)$ \\
Total & $36(100 \%)$ & $36(100 \%)$ \\
\end{tabular}

the atropine is stopped and the steroid/ antibiotic reduced at the discretion of the clinician. All patients continue topical steroids for at least 1 month but for no longer than 3 months postoperatively.

\section{Results}

Thirty six eyes of 36 patients fulfilled the entry criteria, with 20 right eyes and 16 left. Twenty of the 36 patients $(56 \%)$ were male. Thirty five of the patients were white Europeans and one originated from the Indian subcontinent. Thirty three eyes had primary open angle glaucoma (POAG), of which five were noted to have narrow (but not closed) angles on gonioscopy, one had pigmentary glaucoma, and two had glaucoma associated with the pseudoexfoliation syndrome. The mean (median) age at surgery was 69.8 (71.5) with a range of 44-85 years. Patients had been diagnosed as having glaucoma a mean (median) of 33.7 (31) months before surgery (range 1-151). Twenty eight $(78 \%)$ of the procedures were performed by an experienced glaucoma specialist (SAV), the remainder being performed by a trainee under the specialist's supervision.

There were no intraoperative complications noted. Four eyes $(11 \%)$ required argon laser suture lysis in the first postoperative week to facilitate drainage. Twelve eyes (33\%) had postoperative hyphaemas, defined as any level of red blood cells in the anterior chamber (AC), on the first postoperative day. All of these cleared rapidly without apparent detriment. Five eyes (14\%) had a shallow AC postoperatively, defined as less than one half preoperative depth on clinical inspection. One of these required surgical reformation and subsequent lens extraction, due to cornea/lens touch.

The mean (SD, median) follow up period of operated eyes was 50.8 months $(16.5,53)$ with a range of 24-75 months. During the follow up period, nine patients have died and four have repeatedly failed to attend. Since filtering surgery, 13 eyes (36\%) have undergone cataract surgery a mean (median) of 31 (28) months later. Visual acuity had decreased a mean (median) of 2.2 (1.0) lines on the

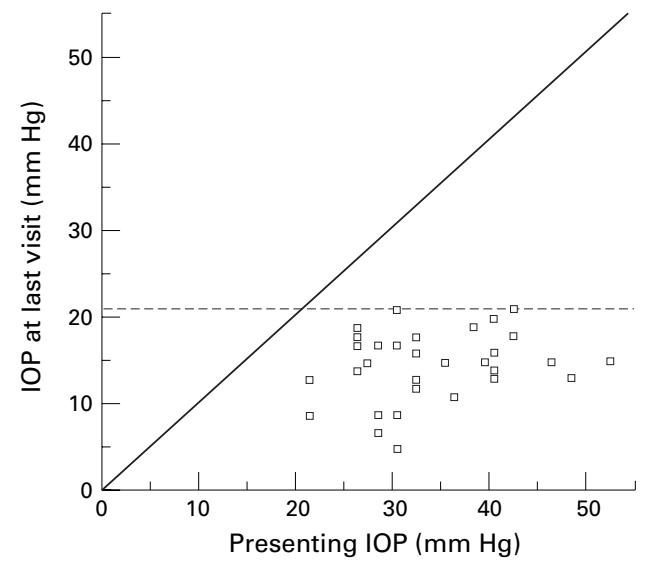

Figure 1 Scattergraph of presenting IOP v IOP at last outpatient visit. Lines of equivalence (solid) and $21 \mathrm{~mm}$ $\mathrm{Hg}$ (broken) shown (some points equate to more than one eye).

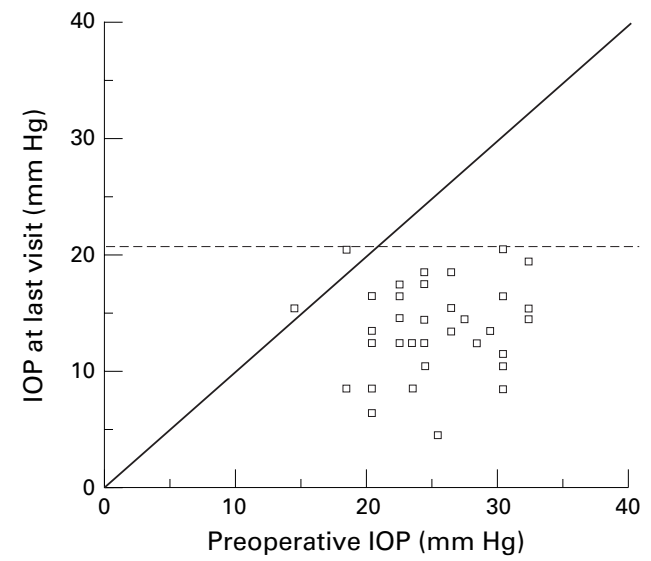

Figure 2 Scattergraph of preoperative IOP $v$ IOP at last outpatient visit. Lines of equivalence (solid) and $21 \mathrm{~mm}$ $\mathrm{Hg}$ (broken) shown (some points equate to more than one eye).

Snellen chart between glaucoma and cataract surgery in these eyes. No eyes have required further surgery to control IOP during the follow up period.

Medications used to control IOP preoperatively and at final follow up are shown in Table 1. The mean (median) number of antiglaucoma medications reduced from 1.4 (1) preoperatively to $0.25(0)$ at the last postoperative visit ( $p<0.0001$ by Wilcoxon test). The eight eyes requiring antiglaucoma medications postoperatively were commenced on treatment a mean (median) of 30 (22.5) months after surgery. The IOP levels before the reintroduction of therapy were $22 \mathrm{~mm} \mathrm{Hg}$ (four eyes), $21 \mathrm{~mm}$ $\mathrm{Hg}$ (three eyes) and $20 \mathrm{~mm} \mathrm{Hg}$ (one eye). Four of the $13(31 \%)$ eyes which went on to have cataract surgery and $4 / 23(17 \%)$ of the remainder required postoperative antiglaucoma medications ( $p=0.47$ by Fisher's exact test).

Table 2 IOPs following microtrabeculectomy

\begin{tabular}{lccccccccc}
\hline & At presentation & Preop & $\begin{array}{l}\text { 6 mon } \\
\text { ths }\end{array}$ & 1 year & 2 years & 3 years & 4 years & 5 years & 6 years \\
\hline Number of eyes & 36 & 36 & 36 & 36 & 36 & 29 & 22 & 15 & 8 \\
Mean IOP (mm Hg) & 33.7 & 24.6 & 11.9 & 12.6 & 13.2 & 13.7 & 13.2 & 12.7 & 12.3 \\
SD & 7.5 & 4.5 & 4.6 & 4.7 & 4.6 & 4.1 & 4.0 & 4.8 & 3.9 \\
Median IOP & 32 & 24 & 12 & 12 & 14 & 14 & 13 & 14 & 11 \\
\hline
\end{tabular}




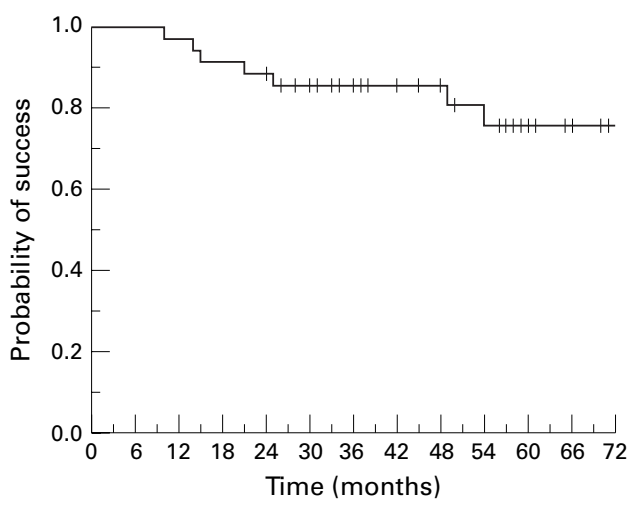

Figure 3 Kaplan-Meier survival analysis considering any IOP recording of $21 \mathrm{~mm} \mathrm{Hg}$ or above as a failure.

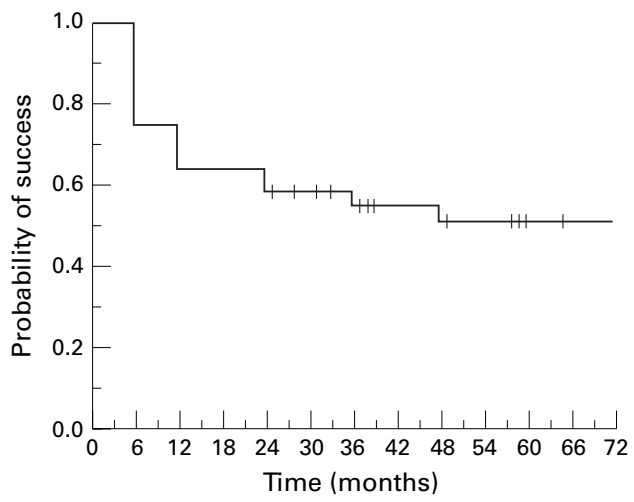

Figure 4 Kaplan-Meier survival analysis considering any IOP recording of $16 \mathrm{~mm} \mathrm{Hg}$ or above as a failure.

The pre- and postoperative IOPs at each time point are shown in Table 2 . Figure $1 \mathrm{dem}-$ onstrates the presenting pressures (the first IOP recorded on presenting to the ophthalmologist) against final recorded IOP. Figure 2 shows preoperative IOPs (on treatment) versus final IOP. Figures 3 and 4 are Kaplan-Meier survival analyses in which any recording of a postoperative IOP $>20 \mathrm{~mm} \mathrm{Hg}$ and $>15 \mathrm{~mm}$ $\mathrm{Hg}$ respectively constitutes a failure of treatment.

All 36 eyes recorded IOPs $<21 \mathrm{~mm} \mathrm{Hg}$ at their last review. A one way ANOVA comparing all postoperative IOPs indicates that there is no significant difference between the IOPs at any of the time points from 6 months to 6 years postoperatively $(\mathrm{F}=0.62, \mathrm{p}>0.05)$.

\section{Discussion}

The first trabeculectomy described by Cairns ${ }^{1}$ involved a $5 \times 5 \mathrm{~mm}$ partial thickness scleral trapdoor and a $4 \times 1 \mathrm{~mm}$ tissue block excision as far as the scleral spur. Our scleral trapdoor at $2 \times 2 \mathrm{~mm}$ is approximately one sixth of the area of the Cairns' procedure, and the $0.75 \times 0.75$ $\mathrm{mm}$ internal opening is just one seventh of the area of the original osteum. Thus the relative sizes of the flap and the internal osteum in "small flap" trabeculectomy are similar to the operation from which it is derived. Such a marked reduction in the size of the surgical incisions might be expected to result in an increased rate of "filtration failure" with time as has been found to occur following holmium laser $\mathrm{ab}$ externo laser sclerostomy. ${ }^{12}$ The results of this study indicate that reducing the size of the procedure does not, however, appear to lead to an increased medium to long term failure rate. This reassuring fact permits the surgeon to take advantage of the potential benefits of a procedure which occupies a smaller area on the surface of the eye.

We consider that there would be sufficient space for up to three small flap trabeculectomies around the superior limbus should reoperation be necessary. This feature is likely to be of particular advantage when performing surgery on eyes that have had previous drainage and/or cataract surgery. Indeed, over the past 7 years, we have found that a microtrabeculectomy can often be performed in the small circumference of "virgin" conjunctiva at the limbus when a traditionally sized procedure would have to be placed inferiorly. In addition, with the use of a smaller scleral trapdoor, the scleral sutures are situated more anteriorly, allowing for easier laser suture lysis should this be necessary.

We acknowledge that true success in glaucoma management should be measured in terms of visual function. However, postoperative IOP control serves as a useful point of comparison with other studies when considering surgical success rates. IOP remains relatively stable between 1 and 4 years following conventional trabeculectomy, ${ }^{13}$ and we have demonstrated that the same is true for small flap trabeculectomy. The mean IOPs in this study tend to be slightly lower than those quoted by other authors using larger scleral flaps. ${ }^{43-15}$ This may be as a result of our relatively low risk patient population or from a combination of our surgical technique and postoperative management.

Migdal et al have reported a $98 \%$ success rate (defined as IOP $<23 \mathrm{~mm} \mathrm{Hg}$ off medication) at 1-5 years following conventional trabeculectomy performed as a primary procedure in POAG. ${ }^{16}$ Only one of our eyes could be considered to have had primary surgery; topical medications, shown to be a risk factor for surgical failure, ${ }^{16}$ having been used in our patients for a mean of nearly 3 years before surgery. Indeed, if we had used the same criteria for "failure" as the Moorfields group (IOP $>22 \mathrm{~mm} \mathrm{Hg}$ on two occasions), none of our eyes would be classified as "failures" at any stage. We do not know, however, whether our eyes deemed to require additional medical treatment would have recorded higher IOPs if treatment had not been instituted.

Our results can, in addition, be compared with those of Nouri-Mahdavi and coworkers ${ }^{17}$ whose preoperative mean IOP was similar to ours (24.1 $\mathrm{mm} \mathrm{Hg}$ versus $24.6 \mathrm{~mm} \mathrm{Hg}$ ) and who also used life table analysis to describe their outcomes. Our survival rates of $80 \%$ at 4 years and $75 \%$ at 5 years (considering an IOP of $21 \mathrm{~mm} \mathrm{Hg}$ and above as a failure) are very similar to those from the New Haven group (83\% and $80 \%)$.

With an increased emphasis on the attainment of a "target pressure", the second of our life table analyses (Fig 4) with a particularly strict criterion for failure (any recorded IOP 
$>15 \mathrm{~mm} \mathrm{Hg}$ ) is interesting. It indicates that $50 \%$ of eyes satisfying our entry criteria will be likely to achieve this level of success over the first 6 years following surgery. To our knowledge, no previous report of unenhanced filtering surgery has used a Kaplan-Meier analysis at this level of IOP.

A hyphaema rate of $33 \%$ is not excessive when compared with previous reports, ${ }^{13}{ }^{18}$ in which rates vary from $7 \%$ to $53 \%$. In our study, any fluid level of blood in the anterior chamber was recorded as hyphaema, and none was the cause of significant morbidity. We consider that the use of the Kelly punch to clean cut a smaller, more anterior block of tissue does reduce the likelihood and volume of any intraoperative haemorrhage. This is in accordance with the work of Konstas and Jay who employed a similar site for their internal sclerostomy. ${ }^{19}$

It is of note that we did not exclude eyes with narrow drainage angles from this series. Of the five eyes to record a shallow AC postoperatively, only one required reformation of the anterior chamber and subsequent cataract surgery. Therefore, the rate of shallow AC when small flap trabeculectomy is performed or supervised by a surgeon experienced in the technique appears less than the first report of trabeculectomy ${ }^{1}$ and similar to other reports of filtering surgery. ${ }^{56}$ We believe the practice of routinely using a limbus based flap and inflating the eye via a paracentesis with both fluid and air at the close of surgery plays an important role in reducing shallow ACs in the early postoperative period.

Thirteen eyes $(36 \%)$ went on to subsequent cataract surgery during our follow up period. This appears high when compared with some reports ${ }^{20}$ but not with others. ${ }^{17} \mathrm{Re}$-examination of the medical records of these patients revealed that $8 / 13$ had acuity reduced to $20 / 40$ or less from cataract before filtering surgery and in five of these the recorded acuity at the time of cataract surgery was within one Snellen line of the prefiltration acuity. We consider that a major factor in the rate of cataract surgery following filtering surgery in this series is the introduction of phacoemulsification in our unit. This has lowered our threshold for intervention in such cases and eyes with significant cataract before filtering surgery are now often scheduled for phacotrabeculectomy.

Although not formally studied in this group of patients, we suspect that by reducing the size of the surgical field we will induce less corneal astigmatism. This in turn may lead to more rapid visual rehabilitation. Initial results in a different cohort of eyes appear promising ${ }^{21}$ and further work is ongoing in our unit on this aspect of the procedure.

We have demonstrated that "small flap" trabeculectomy in a group of "low risk" eyes controls IOP in the medium to long term at least as well as previous reports utilising the conventional full sized procedure. Similar results, without the use of antimetabolites, may not be obtainable in higher risk eyes as the only demonstrable risk factor for failure in our cohort was the prior use of topical medications. ${ }^{14}$ Our results are, however, comparable with those from a similar population undergoing primary filtering surgery with the conventional procedure. ${ }^{16}$

1 Cairns JE. Trabeculectomy: preliminary report of a method. Am f Ophthalmol 1968;66:673-9.

2 Watson PG. Surgery of the glaucomas. $\mathrm{Br} f$ Ophthalmol 1972;56:299-318.

3 Krasnov MM. A modified trabeculectomy. Ann Ophthalmol 1974;6:178-82.

4 Sanders R, MacEwan CJ, Haining WM. Trabeculectomy: effect of varying the surgical site. Eye 1993;7:440-3.

5 Schuster R, Krupin T, Kolker AE, et al. Limbus $\mathrm{v}$ fornix-based conjunctival flap in trabeculectomy: a long
term randomised study. Arch Ophthalmol 1984;102:361-2.

6 Dellaporto A. Experience with trephanotrabeculectomy. Dellaporto A. Experience with trephanotrabeculectom
Trans Am Acad Ophthalmol Otolaryngol 1975;79:362-71.

7 Smith BF, Schuster H, Seidenberg B. Subscleral Smith BF, Schuster H, Seidenberg B. Subscleral
sclerectomy: a double flap operation for glaucoma. $A m \mathcal{F}$ sclerectomy: a double flap

8 Tomey KF, Shamas IV. Single snip trabeculectomy using a specially designed punch. Ophthalmic Surg 1986;17:81618.

9 Khaw PT, Migdal CS. Current techniques in healing modulation in glaucoma surgery. Curr Opin Ophthalmol 1996;72: 24-33.

10 Lanigan L, Sturmer J, Baez KA, et al. Single intraoperative applications of 5-fluorouracil during filtration surgery: early results. Br f Ophthalmol 1994;15:33-7.

11 Vernon SA, Spencer AF. Intraocular pressure control following microtrabeculectomy. Eye 1995;9:299-303.

12 Iwach AG, Hoskins HD Jr, Drake MV, et al. Update of the subconjunctival THC:YAG (holmium) laser sclerostomy ab externo clinical trial: 30 month report. Ophthalmic Surg 1994;25:13-21.

13 Mills KB. Trabeculectomy: a retrospective long term follow up of 444 cases. Br $\mathcal{F}$ Ophthalmol 1981;65:790-5.

14 Lavin MJ, Wormald RPL, Migdal CS, et al. The influence of prior therapy on the success of trabeculectomy. Arch Ophthalmol 1990;108:1543-8.

15 Starita RJ, Fellman RL, Spaeth GL, et al. Effect of varying the site of scleral flap and corneal block on trabeculectomy. Ophthalmic Surg 1984;15:484-7.

16 Migdal C, Gregory W, Hitchings R. Long-term functional outcome after early surgery compared with laser and medicine in open-angle glaucoma. Ophthalmology 1994;101: 1651-6.

17 Nouri-Mahdavi K, Brigatti L, Weitzman M, et al. Outcomes of trabeculectomy for primary open angle glaucoma. Ophthalmology 1995;102:1760-9.

18 Zaidi AA. Trabeculectomy: a review and 4 year follow up. $\mathrm{Br}$ 7 Ophthalmol 1980;64:436-9.

19 Konstas AGP, Jay JL. Modification of trabeculectomy to avoid postoperative hyphaema: the 'guarded anterior fistula' operation. Br F Ophthalmol 1992;76:353-7.

20 Seah KL, Prata JA, Minckler DS, et al. Visual recovery after trabeculectomy. F Glaucoma 1995;4:228-34.

21 Vernon SA, Potgieter F, Zambarakji HJ, et al. Changes in manual and simulated keratometry one and three months following microtrabeculectomy. Invest Ophthalmol Vis Sci 1997;38:S1062. 